Original Research Article

\title{
Liquid dish wash solution - can it be an alternative in future for the expensive and hazardous xylene in hematoxylin and eosin staining of paraffin sections
}

\author{
Swaroop Raj B V ${ }^{1}$, Divya $C^{2}$, Harendra Kumar M.L. ${ }^{3}$ \\ ${ }^{1}$ Dr. Swaroop Raj B.V., Assistant Professor, Department of Pathology, Sri Devaraj Urs Medical College, ${ }^{2}$ Dr. Divya C, \\ Assistant Professor, Department of Anatomy, Sri Devaraj Urs Medical College, ${ }^{3}$ Dr. Harendra Kumar ML, Professor, \\ Department of Pathology, Sri Devaraj Urs Medical College, Tamaka, Kolar, Karnataka, India.
}

Corresponding Author- Dr. Swaroop Raj B.V., Assistant Professor, Pathology, Sri Devaraj Urs Medical College, Tamaka, Kolar, Karnataka. Email: dr.swaroop.raj@gmail.com

\begin{abstract}
Introduction: Xylene is the most commonly used clearing agent as it has excellent deparaffinising and clearing properties. Commercial xylene is a clear, colourless liquid that has a sweet and aromatic odour. However, xylene is not only expensive but also has detrimental effect on human health such as hepatitis, chemical pneumonitis, depression, anaemia, etc. Hence in this study we are replacing xylene with commercially available liquid dish wash solution(LDW) which is not only cheap but also non biohazardous. Aims: To assess the efficacy of LDW with xylene as a deparaffinising and clearing agent in routine hematoxylin and eosin staining (H\&E). Materials and Methods: Blocks from 20 consecutively submitted samples at RL Jalappa Hospital between Jan - June 2015 each of breast, Cervix, Lymphnode, Fat, Salivary Gland, Muscle and Alimentary Tract was taken and two sections of 4-micron thickness were cut and deparaffinised using Liquid Dish Wash and Xylene respectively followed by H \& E Staining. The 280 sections of the 140 blocks were evaluated and scored by two pathologists independently for nuclear staining, cytoplasmic staining, uniformity, clarity and crispness. Results: In the samples evaluated LDW seems to be on par with xylene cleared sections in terms of nuclear and cytoplasmic detail, clarity and crispness. However Xylene cleared sections showed slightly better uniform staining. However both sections were equally good for reporting. Conclusion: Liquid dish wash solution is an excellent alternative to xylene in H\&E Staining. However more extensive studies need to be done on other types of tissues for validation
\end{abstract}

Key words: Xylene, Alternative, Liquid Dish wash, Staining, Xylene free staining.

\section{Introduction}

Histopathology is the gold standard for diagnosis in most surgical cases. Histopathology diagnosis requires tissues to be fixed and processed followed by section cutting and staining. Xylene is the most commonly used clearing agent as it has excellent deparaffinising and clearing properties. Commercial xylene is a clear, colourless liquid that has a sweet and aromatic odour.

However, xylene is not only expensive but also has detrimental effect on human health such as hepatitis, chemical pneumonitis, depression, anaemia, etc [1]. Western countries have strict guidelines regarding the exposure monitoring and maximum exposure limit of chemicals like xylene with strict chemical disposal rules

Manuscript received: $25^{\text {th }}$ March 2018

Reviewed: $4^{\text {th }}$ April 2018

Author Corrected: $10^{\text {th }}$ April 2018

Accepted for Publication: $16^{\text {th }}$ April 2018 for laboratories. In developing countries like ours where cost of reports are kept to a minimum, monitoring and disposal of harmful chemicals like xylene may not be practical and hence safer alternatives for such chemicals are always being researched. Haematoxylin and Eosin (H\&E) Staining has stood the test of time and is used most frequently in surgical pathology. In staining paraffin embedded sections, xylene is used which is hazardous to the working environment.

Many alternatives to xylene such as alcohols, kerosene, mineral oils and lemon water have been tried as an alternative, however standardisation of those reagents and easy availability haven't been possible. Liquid dish wash solution is eco-friendly, cost effective and time effective [2]. Liquid dish wash with its limonene properties and with boiling can dissolve wax. Xylene 
free sections have been reported in earlier studies to be good as or better than conventional section [3-5]. Though xylene is an excellent deparrafinising and clearing agent. Lacunae in knowledge and need for study is to create a safe working environment in laboratory by finding cost effective, non toxic alternatives to chemicals like xylene which requires stringent concentration monitoring and safe disposal. Hence in this study xylene was replaced by $1.7 \%$ commercially available Vim liquid dish wash solution (LDW) which is not only cheap and effective but also non biohazardous.

Existing knowledge: It is well known that xylene though an excellent clearing and deparaffinising agent is hazardous to health and regulation deem it necessary to be monitored and disposed as per strict protocol. In developing countries where strict vapour monitoring, duration of work timings and safe disposal are not followed due to cost constraints.

\section{Objectives}

1. To assess the quality of staining in xylene free sections using $1.7 \%$ liquid dish wash solution.

2. To compare the quality of staining of xylene free staining versus the conventional staining using xylene

\section{Materials and Methods}

Place and Type of Study: It was a laboratory exploratory study conducted at Department of Pathology, R L Jalappa Hospital attached to Sri Devaraj Urs Medical College. Institutional ethical clearance was taken from ethical committee.

Sample Size: Sample size was estimated based on the values obtained from the study by Anuradha Ananthaneni et al, Diagnosis of lesion in conventional $\mathrm{H} \& \mathrm{E}$ was $100 \%$ and in Xylene free H \& E was 95\% [6]. Using this values sample size was estimated by using the formula of difference in proportions. Sample size of $190+90=380$ was obtained at $\alpha=0.05, \beta$ at 0.20 (Power at 80\%).

Sampling Methods- Blocks from 20 consecutively submitted samples at RL Jalappa Hospital after 1st Jan 2015 each of breast, cervix, lymphnode, fat, salivary gland, muscle salivary gland, muscle, thyroid, skin, alimentary tract, liver and kidney was taken. Exclusion criteria was tissues not belonging to the abovementioned specimen categories. Two sections of 4 micron thickness will be cut and deparaffinised using

\section{Original Research Article}

1.7\% commercially available Vim Liquid Dish Wash and Xylene respectively followed by $\mathrm{H} \& \mathrm{E}$ Staining. $1.7 \%$ Liquid Dishwash solution was prepared by diluting $25 \mathrm{ml}$ of Vim Liquid Dish wash in $1500 \mathrm{ml}$ of distilled water which was prepared freshly. The 400 sections of the 200 blocks will be evaluated and scored by two pathologists independently for nuclear staining, cytoplasmic staining, uniformity, clarity and crispness. Scoring system used was the one used by Ankle MR et al [4]. Procedure for staining using Liquid dish wash solution was from a study by Falkeholm et al [3].

Staining protocol has been mentioned below

\section{Conventional Staining}

1. Slide on hot plate / Incubator for $30 \mathrm{mins}$

2. 3 changes of Xylene -3 mins each

3. Absolute Alcohol - $2 \mathrm{~min}$

4. $90 \%$ Alcohol - $2 \mathrm{~min}$

5. $80 \%$ Alcohol $-2 \mathrm{~min}$

6. $70 \%$ Alcohol - $2 \mathrm{~min}$

7. Distilled Water $-5 \mathrm{~min}$

8. Staining with Hematoxylin and Eosin

9. $70 \%$ Alcohol - 2min

10. $80 \%$ Alcohol - $2 \mathrm{~min}$

11. $90 \%$ Alcohol - $2 \mathrm{~min}$

12. Absolute Alcohol - $2 \mathrm{~min}$

13. 3 changes of Xylene -3 minseach

14. Mount with DPX and cover slip

\section{Xylene Free Staining Using Liquid Dish Wash Solution}

1. Slide on hot plate / Incubator for 30 mins

2. 2 changes of $1.7 \%$ Liquid Dish wash Solution in Water bath at $90 \mathrm{C}-30 \mathrm{sec}$

3. Water at $90 \mathrm{C}-15 \mathrm{sec}$

4. 2 changes of tepid water- 15 sec each

5. Staining with Hematoxylin and Eosin

6. Dry in incubator at $60 \mathrm{C}-10 \mathrm{~min}$

7. Mount with DPX and cover slip

\section{Scoring System}

Nuclear Staining: Adequate - Score 1 Inadequate Score 0

Cytoplasmic Staining: Adequate - Score 1 Inadequate Score 0

Clarity: Present - Score 1, Absent - Score 0 
Uniformity of Staining: Present - Score 1, Absent Score 0

Crispness: Present - Score 1, Absent - Score 0

Total score of less than or equal to two was considered inadequate for diagnosis and 3 to 5 was considered adequate for diagnosis.

\section{Original Research Article}

Statistical Analysis- Percentage for individual component scoring and sensitivity and specificity of Xylene free staing was calculated. $P$ value was calculated. Agreement between scoring by two pathologists was assessed \& Cohens Kappa was calculated.

\section{Results}

Table-1: Comparison of Hematoxylin and Eosin Staining Characteristics of various section after conventional staining and xylene free staining using liquid dish wash solution.

\begin{tabular}{|c|c|c|c|c|c|c|}
\hline & & \multicolumn{2}{|c|}{ Conventional Staining } & \multicolumn{2}{|c|}{ Xylene Free Staining } & \multirow[t]{2}{*}{ P Value } \\
\hline & & $\mathbf{n}$ & $\%$ & $\mathbf{n}$ & $\%$ & \\
\hline \multirow{2}{*}{$\begin{array}{c}\text { Nuclear Staining } \\
\text { (Percentage of cases) }\end{array}$} & Adequate & 192 & 96 & 195 & 97.5 & \multirow[t]{2}{*}{$>0.05$} \\
\hline & Inadequate & 8 & 4 & 5 & 2.5 & \\
\hline \multirow{2}{*}{$\begin{array}{l}\text { Cytoplasmic Staining } \\
\text { (Percentage of cases) }\end{array}$} & Adequate & 194 & 97 & 187 & 93.5 & \multirow[t]{2}{*}{$>0.05$} \\
\hline & Inadequate & 6 & 3 & 13 & 6.5 & \\
\hline \multirow{2}{*}{$\begin{array}{c}\text { Clarity } \\
\text { (Percentage of cases) }\end{array}$} & Adequate & 184 & 92 & 188 & 94 & \multirow[t]{2}{*}{$>0.05$} \\
\hline & Inadequate & 16 & 8 & 12 & 6 & \\
\hline \multirow{2}{*}{$\begin{array}{l}\text { Uniformity of staining } \\
\text { (Percentage of cases) }\end{array}$} & Adequate & 184 & 92 & 174 & 87 & \multirow[t]{2}{*}{$>0.05$} \\
\hline & Inadequate & 16 & 8 & 26 & 13 & \\
\hline \multirow{2}{*}{$\begin{array}{c}\text { Crispness } \\
\text { (Percentage of cases) }\end{array}$} & Adequate & 168 & 84 & 164 & 82 & \multirow[t]{2}{*}{$>0.05$} \\
\hline & Inadequate & 32 & 16 & 36 & 18 & \\
\hline \multirow{2}{*}{$\begin{array}{l}\text { Adequate for diagnosis } \\
\text { (Score : } 3-5)\end{array}$} & Adequate & 194 & 97 & 193 & 96.5 & \multirow[t]{2}{*}{$>0.05$} \\
\hline & Inadequate & 6 & 3 & 7 & 3.5 & \\
\hline
\end{tabular}

Significant reduction in staining time was noted between conventional and xylene free staining. Conventional staining using xylene for deparafinisation and clearing took around 55-60 minutes whereas Xylene free staining using LDW took only 25-30 minutes. Hence using xylene free staining reduces the deparaffinisation time by 30 minutes. Average time taken for conventional staining was 64 minutes and for xylene free staining was 28 minutes. 96.5\% cases were adequate for diagnosis (Total Score: 3-5) and 3.5\% was inadequate for diagnosis in xylene free staining versus $97 \%$ cases adequate for diagnosis and 3\% inadequate for diagnosis conventional staining. Nuclear staining, cytoplasmic staining, crispness, uniformity of staining and clarity was $96 \%$ and $97.5 \%, 97 \%$ and $93.5 \%, 92 \%$ and $94 \%, 92 \%$ and $87 \%$ and $84 \%$ and $82 \%$ in Conventional and Xylene Free Staining respectively. The sensitivity and specificity was more than $95 \%$ for xylene free staining. $\mathrm{P}$ value was $>0.05$ showing no significant difference in the two staining techniques. Most parameters showed comparable results between conventional and xylene free staining. However xylene free staining had lower score in uniformity of staining which was usually due to incomplete deparaffinisation which was either due to lower temperature or shorter duration of sections in water bath with liquid dish wash solution. Cohen's Kappa measure of agreement was used to calculate the agreement between the two pathologists in scoring and a value of 0.74 was obtained indicating substantial (good) agreement.

\section{Discussion}

Xylene is used extensively in histopathology laboratories since decades. However concerns are being raised due to xylene substitutes. Xylene is hazardous to the health of health care professionals exposed to it and the severity of health hazards depends amount and duration of exposure to the chemical. New regulations in countries like US by Occupational Safety and Health Administrations and American Conference of Governmental Industrial Hygiene have given limits for exposure and disposal of hazardous chemicals like xylene [7]. Xylene on exposure can cause reactions between mild skin irritation to neurotoxicity [9]. As such measures like chemical monitoring and disposal are not practical in cost cutting in developing countries like ours and hence ecofriendly alternatives have been looked for to be an alternate for xylene. Falkeholm et alwas the first to use liquid dish wash an alternative to the hazardous xylene [3]. Liquid dish wash solution is used in our kitchen to clean utensils due to its detergent and mild acidic action. Liquid dishwash solution is nonbiohazardous, ecofriendly and cheap but also a good deparaffinising agent acting by its detergent action by reducing the 


\section{Original Research Article}

surface tension. It has many advantages such as it is non toxic, not combustible, has an aromatic odor, reduces turn around time and is cost effective. There was almost no difference in the staining using conventional and xylene free techniques when adequacy for diagnosis was considered. Similar results were seen in other studies.

Table-2: Comparison of hematoxylin and eosin staining characteristics of various studies with present study

\begin{tabular}{|c|c|c|c|c|c|c|}
\hline & & $\begin{array}{c}\text { Present } \\
\text { Study }\end{array}$ & $\begin{array}{c}\text { Ankle M } \\
\text { R et al }\end{array}$ & $\begin{array}{c}\text { Anuradha } \\
\text { A et al }\end{array}$ & $\begin{array}{c}\text { Ramulu } \\
\text { S et al }\end{array}$ & $\begin{array}{c}\text { Pinki } \\
\text { Pandey et al }\end{array}$ \\
\hline $\begin{array}{c}\text { Nuclear Staining } \\
\text { (Percentage of cases) }\end{array}$ & Conventional Staining & 96 & 96.6 & 100 & 94 & 83 \\
\cline { 2 - 7 } & Xylene Free Staining & 97.5 & 98.3 & 95 & 96 & 90 \\
\hline $\begin{array}{c}\text { Cytoplasmic Staining } \\
\text { (Percentage of cases) }\end{array}$ & Conventional Staining & 97 & 93.3 & 100 & 92 & 76 \\
\cline { 2 - 8 } & Xylene Free Staining & 93.5 & 83.3 & 100 & 86 & 90 \\
\hline \multirow{2}{*}{$\begin{array}{c}\text { Clarity } \\
\text { Percentage of cases) }\end{array}$} & Conventional Staining & 92 & 85 & 100 & 94 & 86 \\
\cline { 2 - 8 } & Xylene Free Staining & 94 & 88.3 & 100 & 96 & 90 \\
\hline $\begin{array}{c}\text { Uniformity of } \\
\text { staining }\end{array}$ & Conventional Staining & 92 & 70 & 65 & 92 & 88 \\
\cline { 2 - 8 } (Percentage of cases) & Xylene Free Staining & 87 & 50 & 75 & 80 & 72 \\
\hline \multirow{2}{*}{$\begin{array}{c}\text { Crispness } \\
\text { (Percentage of cases) }\end{array}$} & Conventional Staining & 84 & 76.6 & 100 & 96 & 74 \\
\cline { 2 - 8 } & Xylene Free Staining & 82 & 83.3 & 95 & 88 & 95 \\
\hline $\begin{array}{c}\text { Adequate for diagnosis } \\
\text { (Score : 3-5) }\end{array}$ & Conventional Staining & 97 & 88.3 & 100 & 94 & 84 \\
\cline { 2 - 7 } & Xylene Free Staining & 96.5 & 90 & 95 & 90 & 86 \\
\hline
\end{tabular}

Nuclear staining is very important in routine staining as it helps to distinguish between benign and malignant lesions as identifying features such as nucleoli, hyperchromasia, irregular nuclear membrane and mitotic figures favour a diagnosis of malignancy. In our study comparable or better nuclear staining was noted in xylene free staining compared to conventional staining. Cytoplasmic staining, clarity and crispness of staining was comparable between the two staining techniques. Uniformity of staining was slightly better in conventional staining compared to xylene free staining. This has been observed in previous studies by Ramulu S et al, Pinkey Pandey et al, Anuradha A et al and Ankle MR et al [2,4,5,6].

This non - uniformity in staining could be attributed to inadequate deparaffinisation, section tears, thick sections and moisture in sections [8]. Other studies have also had similar shortcomings in the xylene free technique where if temperature and timings of deparaffinisation if not followed may lead to incomplete deparaffinisation leading foci of unstained areas on the slide. Few studies used progressive staining, however we used the regressive staining like few other studies using Harris hematoxylin [2]. Few studies noted a bluish tinge in xylene free, however the present study did not show any such findings. Xylene free staining provided satisfactory results as can be seen by all the studies [9]. It not only provides a safe working environment for laboratory but also decreases the turn around time. However xylene free technique is a temperature sensitive procedure and hence extreme care should be taken regarding the temperature and duration of sections in liquid dish wash solution. In cases of increased time in water bath with liquid dish wash solution the sections would float off whereas reduced time would lead to incomplete dewaxing. There have been occasional reports of mild allergic reactions of skin with use of liquid dish wash solution in laboratory personnel [11]. There have been similar mild reactions with other xylene substitutes that have been used [12]. At the end of the study a feedback was taken from the technicians regarding the use of xylene free staining. Though initially hesitant to use the xylene free technique after knowing the health hazards of xylene and the reduced turn around time and comparable staining characteristics of xylene free technique, the technicians were not only willing but wanted to adopt the xylene free technique for routine histopathology.

\section{Conclusion}

As a pathologist as important as it is to give a correct diagnosis, it is to provide a safe working environment to healthcare professionals working at the laboratory.

Liquid dish wash solution is an excellent alternative to xylene free sections as it is not only ecofriendly and non-biohazardous but also effective deparaffinising agent and less time consuming as shown by the results above. However care has to be taken temperature maintenance in water bath is very important as lower temperatures lead to inadequate deparaffinisation and non-uniformity of staining. 


\section{What is this study adding to existing knowledge?}

It is well known that xylene is not only expensive but hazardous to health of laboratory personnel who are exposed to it. Several alternatives have been tried and documented but either the procedure is not standardized for all types of tissues or the section quality hasn't been satisfactory when attempted in routine histopathology. This study provides the standardized procedure and documents satisfactory quality of staining of variety of tissues so that the procedure can be adopted in all laboratories easily.

Contribution of each author: Dr Swaroop Raj B V Concept, intellectual ideas, project work and manuscript writing. Dr Divya C - Project work, manuscript editing and fine tuning. Dr Harendra Kumar ML - Manuscript editing, fine tuning and critical review.

Funding: Nil, Conflict of interest: None initiated Permission from IRB: Yes

\section{References}

1. Kandyala R, Raghavendra SP, Rajasekharan ST. Xylene: An overview of its health hazards and preventive measures. J Oral Maxillofac Pathol. 2010 Jan; 14 (1):1-5. doi: 10.4103/0973-029X.64299.

2. Ramulu S, Koneru A, Ravikumar S, Sharma P, Ramesh D, Patil R. Liquid dish washing soap: an excellent substitute for xylene and alcohol in hematoxylin and eosin staining procedure. J Orofac Sci 2012; 4 (1): 37-42.doi: 10.4103/0975-8844.99890.

3. Falkeholm L, Grant CA, Magnusson A, Möller E. Xylene-free method for histological preparation:a multicentre evaluation.LabInvest.2001Sep;81(9):1213-21.

4. Ankle MR, Joshi PS. A study to evaluate the efficacy of xylene free hematoxylin and eosin staining procedure as compared to the conventional hematoxylin and eosin staining: An experimental study.JOMFP 2011;15(2): 161-167. doi: 10.4103/0973-029X.84482.

\section{Original Research Article}

5. Pinki Pandey, Alok Dixit, Aparna Tanwar, Anuradha Sharma, Sanjeev Mittal. A comparative study to evaluate liquid dish washing soap as an alternative to xylene and alcohol in deparaffinization and hematoxylin and eosin staining. J Lab Physicians 2014; 6 (2):84-90. doi: 10.4103/0974-2727.141504.

6. Anuradha Ananthaneni, Srilekha Namala, Vijay Srinivas Guduru, et al., Efficacy of 1.5\% Dish Washing Solution and 95\% Lemon Water in Substituting Perilous Xylene as a Deparaffinizing Agent for Routine $\mathrm{H}$ and E Staining Procedure: A Short Study. Scientifica. 2014; 2014: 11.doi.org/10.1155/ 2014 /707310.

7. Buesa RJ, Peshkov MV. Histology without xylene. Ann Diagn Pathol. 2009 Aug;13(4): 246-56. doi: 10. 1016/j. anndiagpath. 2008.12.005. Epub 2009 Feb 5.

8. Hamil JR, Spencer JM, Morgan MB. Histotechnique and staining troubleshooting. On Atlas of Mohs and Frozen Section Cutaneous Pathology. Ch. 20. New York: Springer Link; 2009.

9. Chen CY, He T, Mao XL, Friis TE, Qin RH, Jian YT. A novel xylene substitute for histotechnology and histochemistry. Biotech Histochem. 2010 Aug; 85 (4): 231-40. doi: 10.3109/10520290903235445.

10. Miller M, Miller D, Driscoll PE. Biodegradable, effective substitute for xylene in the ehrlich indole procedure. J Clin Microbiol 1994;32:2028-30.

11. Foti C, Zambonin CG, Conserva A, et al. Occupational contact dermatitis to a limonene-based in a histopathology technician. Contact Dermat 2007;56 (2): 109-12.

12. Tarbet F, Anderson J. Toxin reduction in Histology. A look at advantages, disadvantages of xylene substitutes. Advance AdmLab 2005;14(4):42.

\section{How to cite this article?}

Swaroop Raj B V, Divya C, Harendra Kumar M.L. Liquid dish wash solution - can it be an alternative in future for the expensive and hazardous xylene in hematoxylin and eosin staining of paraffin sections. Trop J Path Micro 2018;4(2):139143. doi: 10.17511/jopm.2018.i2.03 